\title{
Ex vivo mono-ring technique simplifies culotte stenting for treatment of true bifurcation lesions: Insights from bench testing and clinical application
}

\author{
Lianglong Chen*, Lin Fan*, Yukun Luo*, Wenliang Zhong*, Linlin Zhang,
} Zhaoyang Chen, Chaogui Lin, Yafei Peng, Xingchun Zheng, Xianfeng Dong, Wei Cai

Department of Cardiology, Fujian Medical University Union Hospital,

Provincial Institute of Coronary Artery Disease, Fujian, PR of China

\begin{abstract}
Background: Despite various culotte-based stenting techniques available clinically, the optimal one remains undetermined. The study aimed to test whether ex vivo mono-ring culotte stenting (MRC) was technically feasible and superior to mini culotte stenting (MCS) in treatment of coronary bifurcation lesions.
\end{abstract}

Methods: Mono-ring culotte stenting was characterized by ex vivo wiring of the most proximal cell of the side branch (SB) stent to ensure a mono-ring result of the culotte stenting. Comparison of MRC vs. MCS in treatment of true bifurcation lesions was performed in vitro $(n=15$ for each group) and in clinical case-controlled study with propensity matching at a ratio of $1: 2$ ( $n=21$ for $M R C$ group; $n=42$ for MCS group).

Results: Compared to MCS, MRC had lower incidence of stent under-expansion band (0\% vs. $53.3 \%, p=0.002)$ and less residual ostial area stenosis of $S B(9.2 \pm 9.0 \%$ vs. $20.0 \pm$ $\pm 14.8 \%, p=0.023)$, as assessed in vitro by micro-computed tomography. In a case-controlled study, no adverse cardiac events were observed in the MRC group. The procedural success was similar between MRC and MCS (100\% vs. 95.2\%, $p=0.548)$, but MRC had less residual ostial stenosis of the $S B(8.7 \% \pm 11.0 \%$ vs. $16.8 \% \pm 11.2 \%, p=0.008)$, lower procedural (33.3 \pm 9.5 min vs. $46.7 \pm 15.6 \mathrm{~min}, p=0.001)$ and fluoroscopic $(19.7 \pm 4.9 \mathrm{~min}$ vs. $26.2 \pm 7.1 \mathrm{~min}, p<0.001)$ time, and less contrast use $(114.3 \pm 28.9 \mathrm{~mL} v \mathrm{~s} .156 .5 \pm 56.4 \mathrm{~mL}$, $p=0.002$ ).

Conclusions: Mono-ring culotte stenting as compared to MCS is associated with better bifurcation stent morphology, less procedural complexity and residual ostial SB stenosis. (Cardiol J 2016; 23, 6: 673-684)

Key words: coronary bifurcation lesions, percutaneous coronary intervention, culotte stenting

\footnotetext{
Address for correspondence: Lianglong Chen, MD, PhD, FACC, FESC, or Lin Fan, MD, PhD, 29 Xinquan Road, Fuzhou, China, Postal Code: 350001, tel: +86 13950303022, +86 591 83357896, fax: + 8659183308713 ,

e-mail: lianglongchen@126.com; fanlin1975@126.com

*The first four authors contributed to this work equally.

Received: 17.04.2016 Accepted: 13.07.2016
} 


\section{Introduction}

As firstly introduced by Chevalier et al. [1] for treatment of coronary bifurcation lesions, the culotte stenting underwent several modifications in order to improve the procedural safety and efficacy [2-4]. Nevertheless, the intrinsic procedural complexity, strict requirement of similar branch size and potential risks of acute branch loss, in-stent restenosis, or thrombosis remain the unsolved issues. Modified mini-culotte stenting (MCS) [2], to stent the side branch (SB) first with miniprotrusion of the stent into the main vessel (MV) and to pre-imbed a balloon in the main branch (MB) for preventing branch loss, had lower requirement of similar branch size and was more safe and efficient in treatment of coronary bifurcation lesions. However, if the branch diameter difference (BDD) between MB and SB is too significant, a stent under-expansion band (SUEB) will occur around the polygon of confluence, which will cause stent malapposition around the bifurcated zone and residual obstruction at the ostium of the $\mathrm{SB}$ or $\mathrm{MB}$, likewise increasing risks of in-stent restenosis or thrombosis [4]. In order to lessen SUEB and its related risks, we further modified the MCS to develop the double kissing MCS which was characterized by performing intermediate kissing balloon dilation (iKBD) prior to the MB stenting. Double kissing MCS has been shown to be more efficient in improving bifurcated stent morphology in bench testing and in reducing SB restenosis and target lesion/vessel revascularization in clinical application [4]. Despite this fact, there are numerous culotte-based stenting techniques available currently; the optimal one has not been established yet, particularly, the newly developed culotte techniques remain technically complex and procedurally challenging. Accordingly, we propose a simple culotte stenting technique, the mono-ring culotte stenting (MRC), which is characterized by ex vivo wiring of the most proximal cell of the SB stent as an anchor to ensure a mono-ring result of the culotte stenting.

The present study was to test whether ex vivo mono-ring technique for the culotte stenting was technically feasible, and if MRC was superior to MCS in in vitro and in clinical treatment of coronary bifurcation lesions.

\section{Methods}

\section{Stenting procedure for bench testing} and clinical application

Mini-culotte stenting was performed according to the description in previous studies [2-4]. The major steps of MRC were shown in Figure 1 and described as follows: 1) wiring of the SB and $\mathrm{MB}$, and pre-dilating the branches as necessary; 2) extra-corporeal wiring of the most proximal cell of the SB stent with the hard end of the MB wire (as an anchoring wire) and crossing the SB wire via the central lumen of the SB stent, advancing the $\mathrm{SB}$ stent over the SB wire into the SB until the stent is anchored by the MB wire, then deploying the SB stent; 3) preserving the SB stent balloon and advancing another balloon over the $\mathrm{MB}$ wire to perform a sequential iKBD (inflating SB balloon first with higher pressure, followed by MB balloon inflating with lower pressure); 4) after removal of the two balloons and the SB wire, implanting the $\mathrm{MB}$ stent, and 5) rewiring the $\mathrm{SB}$ at a point close to the carina and then performing a final kissing balloon dilation (fKBD) with the two non-compliant balloons to finish the procedure. Figure 2 shows a representative case sample of bench testing or clinical practice with MRC.

\section{Bench testing}

Experimental protocol. Bench testing was performed on the coronary bifurcation model (Abbott Vascular Temecula), which was made from uniform silicone, according to Murray's law. Testing was repeated 5 times per category of distal bifurcation angulations of $<35^{\circ}, 35-70^{\circ},>70^{\circ}$, resulting in a total of 30 tests, 15 respectively for $\mathrm{MRC}$ and MCS, as shown in the flow chart (Fig. 3). For emulating steps of MRC or MCS and better showing the impact of BDD on the stenting results, open-cell designed stents (Excel ${ }^{\mathrm{TM}}$, JW Medical Inc., Shandong, China; Resolute ${ }^{\mathrm{TM}}$, Metronic Co., Minnesota, USA) of $2.5 \mathrm{~mm}$ and $3.5 \mathrm{~mm}$ were used to stent the SB and the MV/MB, respectively.

Quantitative and qualitative measurements. The stenting procedures were observed visually and recorded with a high-resolution digital video recorder (L-1ex/TT02RX, ELMO, Japan); the final results were examined by micro-computed tomography (mCT) (SkyScan 1176, SkyScan, Belgium).

Quantitative analysis was performed with analytic software for mCT images (control software for SkyScan 1176) to measure the reference stent area (A1), the smallest stent area at the site of the stent-overlapped segment or polygon of confluence (A2), and branch ostial area (A3). Reference stent area for the MV, MB and SB was measured at the MV stent proximal edge, MB stent distal edge and $\mathrm{SB}$ stent distal edge, respectively. Branch ostial area was measured at the ostium of the $\mathrm{MB}$ and $\mathrm{SB}$, respectively. The width of SUEB was defined as the distance from the superior border to inferior border of SUEB, longitudinally. The severity of SUEB, if 


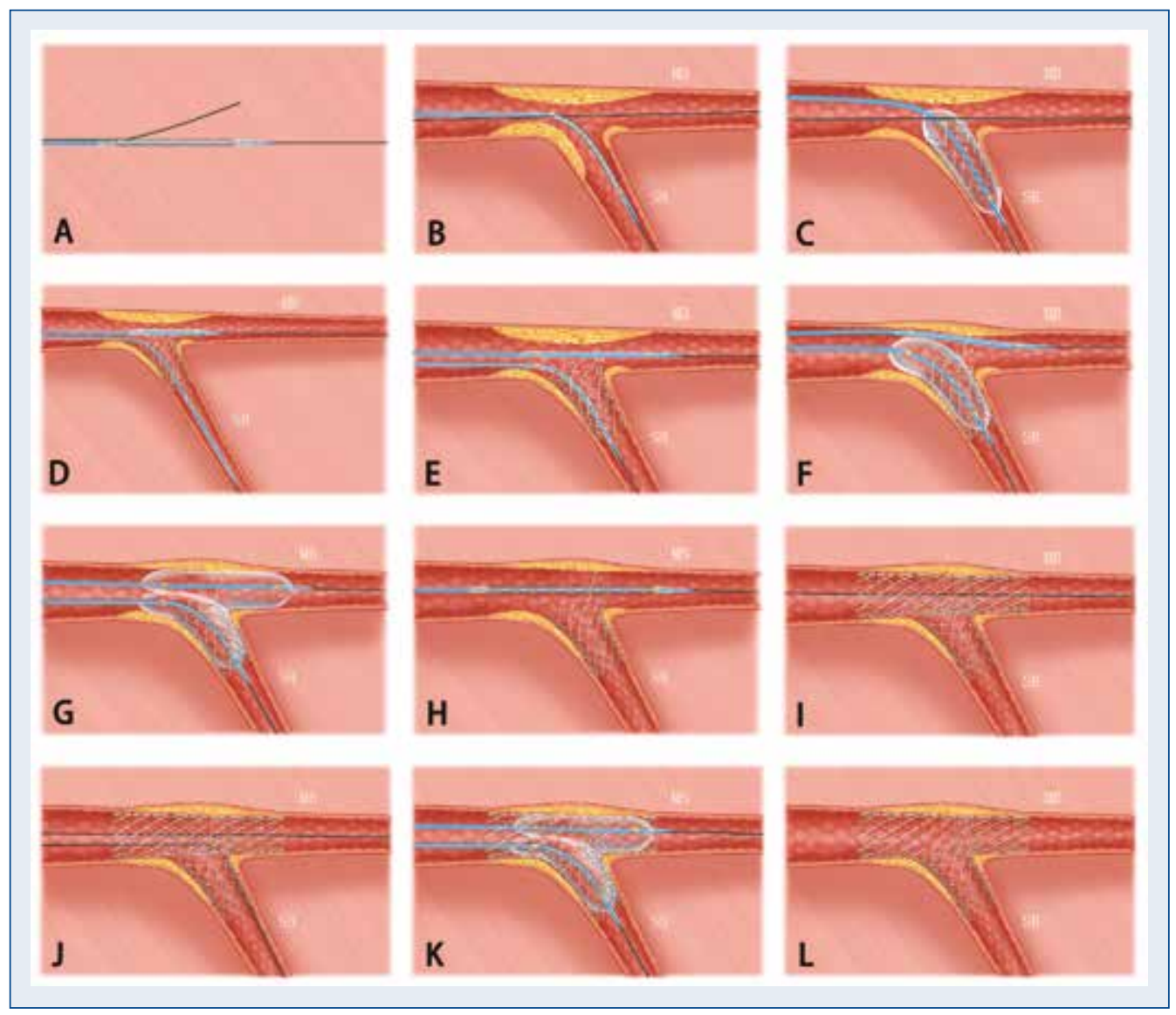

Figure 1. Major steps of mono-ring culotte stenting; A. Ex vivo wiring of the last cell of the side branch (SB) stent with the hard end of the main branch (MB) wire and crossing the SB wire via the central lumen of SB stent; $B$. Pinching the tails of SB and MB wire tightly, then inserting the stent into SB till it is unable to be pushed forward; $C$. Deploying the SB stent; D. Pushing the SB stent balloon deeply and inserting another through the last cell of SB stent; E. Pulling back the SB stent balloon and keeping it at the same level with the MB balloon; F, G. Dilating the SB balloon first and then dilating the MB balloon; H, I. Inserting and deploying the MB stent; J. Rewiring the SB at the point close to the carina; K. Performing final kissing balloon dilation with two non-compliant balloons; L. Final result.

any in the stent-overlapped segment or polygon of confluence, was calculated with (A1 - A2) / $/ \mathrm{A} 1 \times 100 \%$ (Fig. 4). The residual ostial area stenosis (ROAS) for the $\mathrm{SB}$ or MB was calculated with $\left(\mathrm{A}_{\text {for } \mathrm{MB} / \mathrm{SB}}-\mathrm{A} 3_{\text {for ostial MB/SB }}\right) / \mathrm{A} 1_{\text {for MB/SB }} \times 100 \%$, as reported in previous study [5].

Qualitative analysis was performed to assess the final stent configuration based on the videos and $\mathrm{mCT}$ images. A 4-point system was developed for grading the bifurcated stent coverage (Fig. 5). Firstly, the grading region was set in the bifurcated and adjacent area and segmented into polygon of confluence, the carina, and segments $5 \mathrm{~mm}$ distal to the SB or MB ostium. Secondly, for describing stent deformation, the cell distribution was used as a major index: even - similar cell size or the biggest cell size $<1.5$ times of normal cell size; uneven - ir- regular strut distribution or the biggest cell size $=1.5-2.5$ times of normal cell size; uncovered gap - severely irregular strut distribution or the biggest cell size (gap) $>2.5$ times of normal cell size. Finally, bifurcation stent coverage (BSC) was graded by the scoring system: BSC-3 (complete $\mathrm{BSC}$ ), no obvious uneven strut distribution noted in all segments; BSC-2 (slight incomplete BSC), uneven strut distribution noted in at least one segment; BSC-1 (moderate incomplete BSC), significant uneven strut distribution; BSC-0 (severe incomplete BSC), stent-uncovered gaps.

Parameters and definitions. SUEB or ROAS was introduced to reflect stent expansion and BSC score to stent coverage. Usually, SUEB was located in the stent-overlapped segment or polygon of confluence and defined as the stent lumen 


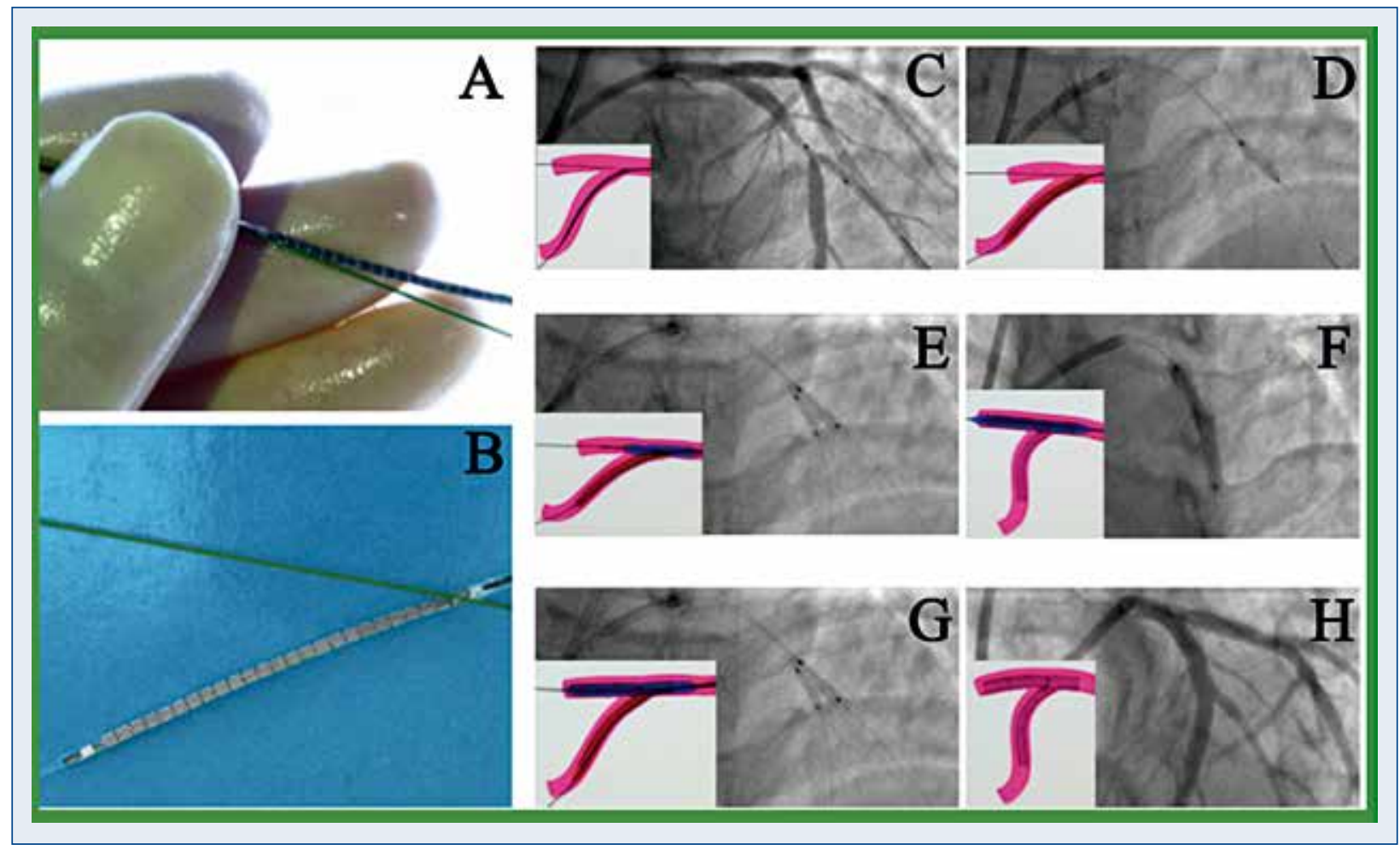

Figure 2A-H. Representative case samples of bench testing and clinical practice with mono-ring culotte stenting.

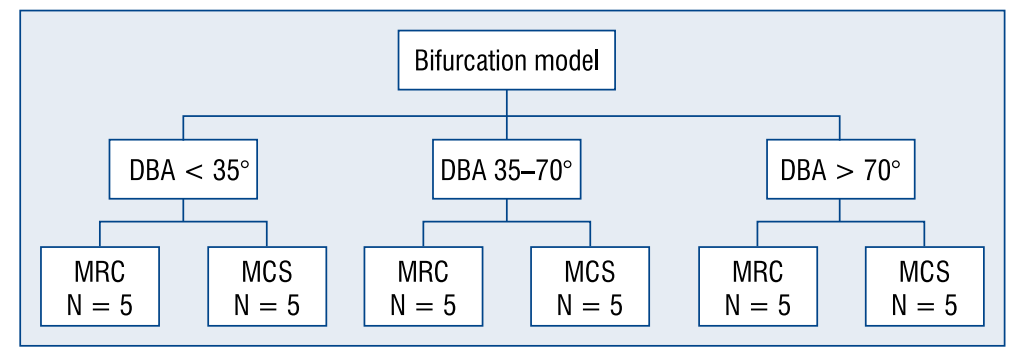

Figure 3. Study flow chart; DBA — distal bifurcation angulations; MCS - mini-culotte stenting; MRC - mono-ring culotte stenting.

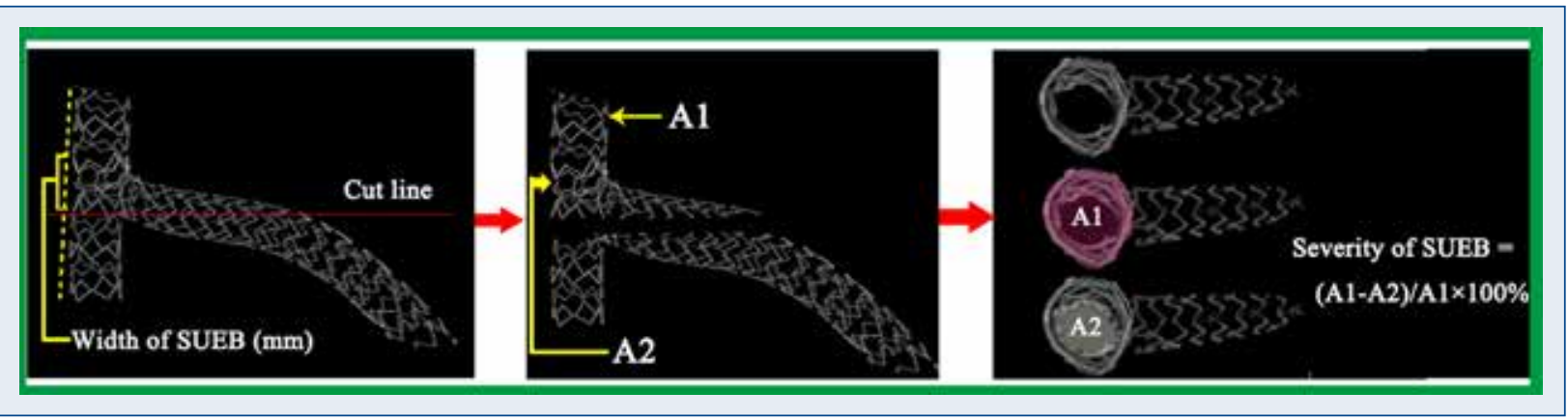

Figure 4. Measurement for stent under-expansion band (SUEB) in vitro. The pictures represent mini-culotte stenting in bench testing. The cut line indicated the stent was cross-sectionally cut open at the inferior border of SUEB; A1 - reference stent area; A2 - the smallest stent area at the site of the stent-overlapped segment or polygon of confluence. 


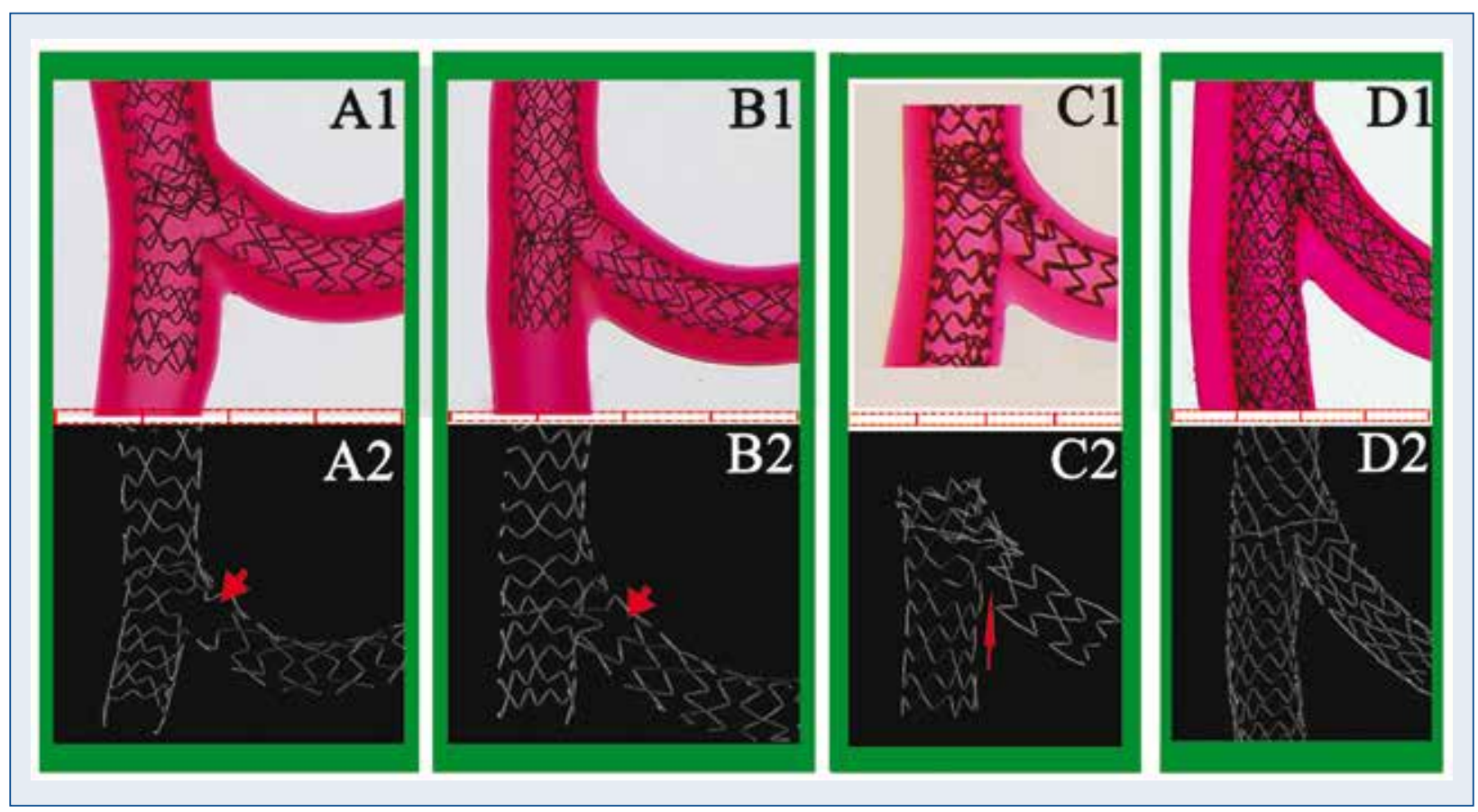

Figure 5. The 4-point scoring method for bifurcation stent coverage. Incomplete bifurcation stent coverage (BSC) was indicated as red arrow; A - severe incomplete BSC scored as 0 ; B - moderate BSC as 1; C - slight incomplete BSC as 2; D - complete BSC as 3 .

under-expansion $\geq 20 \%$, ROAS as the ostial area stenosis $\geq 70 \%$, and incomplete BSC as BSC score $\leq 2$, respectively.

\section{Clinical application}

Patient selection. From June 2014 to June 2015 , patients aged 18 years or older, with true coronary bifurcation lesions and treated with MRC, were considered eligible for enrollment. True coronary bifurcation lesions were referred to Medina's bifurcation lesions (type $1,1,1 ; 0,1,1$; $1,0,1)[6,7]$. The diameter of the MB and SB by visual estimate should be $\geq 2.5 \mathrm{~mm}$ and $\geq 2.25$ $\mathrm{mm}$, respectively. Patients, with ST-elevation acute myocardial infarction within $24 \mathrm{~h}$, life expectancy $<1$ year, or allergy to any of the drugs used (aspirin, clopidogrel, etc.), were excluded. All procedures performed in studies involving human participants were in accordance with the ethical standards of our institutional Research Committee and the 1964 Helsinki Declaration with its later amendments or comparable ethical standards. All patients gave written informed consent.

To test the clinical feasibility of MRC, the patients who underwent MCS from June 2014 to June 2015 were retrospectively screened in historical bifurcation percutaneous coronary intervention database of Fujian Medical University Union hospital. The inclusion and exclusion criteria were the same as for MRC. The eligible patients were used as control group.

Coronary angiography and analysis. Coronary angiography was performed at baseline, at procedural end after intracoronary injection of $200 \mu \mathrm{g}$ nitroglycerin. Quantitative coronary angiographic analysis was performed offline in the MV, MB and SB segments with a validated automatic edge-detection system (QuantCor QCA System, Siemens AG, Erlangen, Germany). In the MV and SB segments, the major parameters of the minimal lumen diameter, percent diameter stenosis and reference vessel diameter were analyzed within the stent and the margins $5 \mathrm{~mm}$ proximal and distal to the stent edge [8].

Events and definitions. The technical or device success was defined as successful implantation of bifurcated stents with completion of fKBD without immediate complications, angiographic success as a minimal stenosis diameter reduction to $<20 \%$ with grade 3 Thrombolysis in Myocardial Infarction (TIMI) flow in both branches postprocedurally. Procedural success was defined as an angiographic success without in-hospital major cardiac adverse events of cardiac death, myocardial 
Table 1. Comparison of the bifurcated stent morphologies.

\begin{tabular}{lccc}
\hline Variables & MRC $(\mathbf{n}=\mathbf{1 5})$ & MCS $(\mathbf{n}=15)$ & $\mathbf{P}$ \\
\hline SUEB: & & & 0.002 \\
Incidence* & $0(0 \%)$ & $8(53.3 \%)$ & $<0.001$ \\
Severity [\%] & $7.9 \pm 4.0$ & $20.5 \pm 5.9$ & $<0.001$ \\
Width [mm] & $0.05 \pm 0.04$ & $1.83 \pm 1.78$ & 0.042 \\
ROAS, SB: & & & 0.023 \\
Incidence* & $0(0 \%)$ & $5(33.3 \%)$ & $20.0 \pm 14.8$ \\
Severity & $9.2 \pm 9.0$ & & 1.000 \\
ROAS, MB: & $0(0 \%)$ & $0.6 \pm 7.8$ & 0.551 \\
Incidence & $4.7 \pm 9.5$ & $4(26.7 \%)$ & 0.651 \\
Severity & $2(13.3 \%)$ & & \\
Incidence of incomplete BSC & & \\
\hline
\end{tabular}

${ }^{*}$ Fisher's exact test; BSC — bifurcated stent coverage; MB — main branch; MCS — mini culotte stenting; MRC — mono-ring culotte stenting; ROAS - residual ostial area stenosis; SB — side branch; SUEB — stent under-expansion band

infarction, target vessel/lesion revascularization or in-stent thrombosis [9].

Non-Q-wave myocardial infarction was defined as cardiac troponin $\mathrm{T} /$ cardiac troponin $\mathrm{I}$ (cTnT/ $/ \mathrm{cTnI}$ ) increase to $\geq 5$ times of the upper reference limit combined with clinical signs and without new onset of pathological Q waves; Q-wave myocardial infarction as new development of pathological $\mathrm{Q}$ waves in two contiguous leads together with clinical signs. Target vessel/lesion revascularization was the repeat target vessel/lesion therapy either by percutaneous coronary intervention or surgery. In-stent thrombosis was diagnosed according to the Academic Research Consortium definition [10].

\section{Statistical analysis}

All analyses were performed using SPSS 20.0 (SPSS Inc., Chicago, USA). Discrete or categorical variables are presented as numbers or percentages, continuous variables as mean \pm standard deviation.

We used a method of propensity score matching to reduce the treatment selection bias and the impact of potential confounding factors from the baseline lesion characteristics. The propensity score was calculated with a logistic regression model without regard to outcome variables. All bifurcation lesion factors that affect outcomes on univariate analysis were considered candidate variables. All variables with $\mathrm{p}<0.20$ were retained. The reliability of the model was evaluated using the Hosmer-Lemeshow test. According to the nearest matching algorithm, we created case-matched pairs without replacement at a 1:2 ratio.
To compare differences of the variables between the two groups, $\chi^{2}$ or Fisher's exact test was employed for the discrete variables and t-test for the continuous variables. All $\mathrm{p}$ values were 2 -sided and the level of significance was $5 \%$.

\section{Results}

\section{Bench testing}

The stent sorts, BDD (3.5 mm stent for MB, $2.5 \mathrm{~mm}$ stent for SB) and branch distal angulation $\left(53.2 \pm 12.9^{\circ}\right.$ for $\mathrm{MRC}$ and $52.9 \pm 10.6^{\circ}$ for MCS, $\mathrm{p}>0.05)$ were similar between MRC and MCS.

Table 1 shows the bifurcated stent morphology of MRC and MCS. Compared with MCS, MRC had lower incidence $(0 \%$ vs. $53.3 \%, \mathrm{p}=0.002)$, less severity $(7.9 \pm 4.0 \%$ vs. $20.5 \pm 5.9 \%, \mathrm{p}<0.001)$ and narrower width $(0.05 \pm 0.04 \mathrm{~mm}$ vs. $1.83 \pm$ $\pm 1.78 \mathrm{~mm}, \mathrm{p}<0.001)$ of SUEB; MRC had also lower incidence $(0 \%$ vs. $33.3 \%, \mathrm{p}=0.042)$ and less severity $(9.2 \pm 9.0 \%$ vs. $20.0 \pm 14.8 \%, \mathrm{p}=$ $=0.023)$ of ROAS in the SB, with similar incidence and severity of ROAS in the MB. Moreover, the incidence of incomplete BSC was infrequent in the MRC group and similar as that of the MCS group (13.3\% vs. $26.7 \%, \mathrm{p}=0.651$ ).

\section{Clinical application}

Eligible patients comprised 23 patients treated with MRC and 119 patients with MCS. Based on a logistic regression analysis, 9 lesion characteristics (Medina classification, SYNTAX score, $\mathrm{BDD}$, the lesion length, minimal lumen diameter and diameter stenosis of $\mathrm{MB}$ and $\mathrm{SB}$ ) were used 
Table 2. Baseline clinical and lesion characteristics.

\begin{tabular}{|c|c|c|c|c|c|c|}
\hline \multirow[t]{2}{*}{ Variables } & \multicolumn{3}{|c|}{ Before propensity score matching } & \multicolumn{3}{|c|}{ After propensity score matching } \\
\hline & $\operatorname{MRC}(n=23)$ & $\operatorname{MCS}(n=119)$ & $\mathbf{P}$ & $\operatorname{MRC}(n=21)$ & $\operatorname{MCS}(n=42)$ & $\mathbf{P}$ \\
\hline Male & $17(73.9 \%)$ & $97(81.5 \%)$ & 0.402 & $15(71.4 \%)$ & $31(73.8 \%)$ & 0.841 \\
\hline Age [years] & $61.7 \pm 10.0$ & $64.1 \pm 9.5$ & 0.278 & $61.7 \pm 10.3$ & $64.2 \pm 11.2$ & 0.391 \\
\hline Hypertension & $12(52.2 \%)$ & $68(57.1 \%)$ & 0.660 & $11(52.4 \%)$ & $21(50.0 \%)$ & 0.859 \\
\hline Diabetes mellitus & $8(34.7 \%)$ & $35(29.4 \%)$ & 0.608 & $8(38.1 \%)$ & $16(38.1 \%)$ & 1.000 \\
\hline Hyper-cholesterol & $9(39.1 \%)$ & $40(33.6 \%)$ & 0.610 & $8(38.1 \%)$ & $15(35.7 \%)$ & 0.853 \\
\hline Current smoking & $7(30.4 \%)$ & $54(45.4 \%)$ & 0.185 & $7(33.3 \%)$ & $15(35.7 \%)$ & 0.852 \\
\hline Prior myocardial infarction* & $4(17.4 \%)$ & $27(22.7 \%)$ & 0.784 & $3(14.3 \%)$ & $6(14.3 \%)$ & 1.000 \\
\hline Prior $\mathrm{PCl}^{*}$ & $2(8.7 \%)$ & $23(19.3 \%)$ & 0.368 & $2(9.5 \%)$ & $5(11.9 \%)$ & 1.000 \\
\hline Clinical presentation*: & & & 0.248 & & & 1.000 \\
\hline Stable angina & $3(13.0 \%)$ & $31(26.1 \%)$ & & $2(9.5 \%)$ & $5(11.9 \%)$ & \\
\hline Unstable angina & $14(60.9 \%)$ & $71(59.7 \%)$ & & $13(61.9 \%)$ & $26(61.9 \%)$ & \\
\hline NSTEMI & $5(21.7 \%)$ & $15(12.6 \%)$ & & $5(23.8 \%)$ & $9(21.4 \%)$ & \\
\hline STEMI & $1(4.4 \%)$ & $2(1.6 \%)$ & & $1(4.8 \%)$ & $2(4.8 \%)$ & \\
\hline LVEF > $50 \% *$ & $19(82.6 \%)$ & $94(72.9 \%)$ & 1.000 & $18(85.7 \%)$ & $36(85.7 \%)$ & 1.000 \\
\hline Lesion location*: & & & 0.541 & & & 1.000 \\
\hline LM/LAD/LAX & $7(30.4 \%)$ & $39(32.8 \%)$ & & $6(28.6 \%)$ & $11(26.2 \%)$ & \\
\hline LAD/Ds & $13(56.5 \%)$ & $72(60.5 \%)$ & & $12(57.1 \%)$ & $24(57.1 \%)$ & \\
\hline LCX/OMs & $2(8.7 \%)$ & $6(5.0 \%)$ & & $2(9.5 \%)$ & $5(11.9 \%)$ & \\
\hline RCA/PLA & $1(4.4 \%)$ & $2(1.7 \%)$ & & $1(4.8 \%)$ & $2(4.8 \%)$ & \\
\hline Medina classifying*: & & & 0.085 & & & 1.000 \\
\hline Type $1,1,1$ & $20(87.0 \%)$ & $74(62.2 \%)$ & & $18(85.7 \%)$ & $36(85.7 \%)$ & \\
\hline Type $0,1,1$ & $2(8.7 \%)$ & $29(24.4 \%)$ & & $2(9.5 \%)$ & $4(9.5 \%)$ & \\
\hline Type 1,0,1 & $1(4.3 \%)$ & $16(13.4 \%)$ & & $1(4.8 \%)$ & $2(4.8 \%)$ & \\
\hline Distal bifurcation angle $>70^{\circ}$ & $7(30.4 \%)$ & $39(32.8 \%)$ & 0.826 & $5(23.8 \%)$ & $10(23.8 \%)$ & 1.000 \\
\hline $\mathrm{BDD}[\mathrm{mm}]$ & $0.68 \pm 0.41$ & $0.51 \pm 0.31$ & 0.025 & $0.74 \pm 0.39$ & $0.65 \pm 0.31$ & 0.249 \\
\hline Calcification* & $3(13.0 \%)$ & $10(8.4 \%)$ & 0.443 & $3(14.3 \%)$ & $6(14.3 \%)$ & 1.000 \\
\hline Tortuosity* & $5(21.7 \%)$ & $16(13.4 \%)$ & 0.305 & $4(19.0 \%)$ & $8(19.0 \%)$ & 1.000 \\
\hline SYNTAX score & $20.7 \pm 7.1$ & $25.5 \pm 10.1$ & 0.031 & $21.4 \pm 7.0$ & $20.2 \pm 6.9$ & 0.510 \\
\hline
\end{tabular}

*Indicated by Fisher's exact test. BDD — branch diameter difference between the main branch and side branch; Ds — diagonal branches; LAD - left anterior descending artery; LCX - left circumflex artery; LM — left main stem; LVEF — left ventricular ejection fraction; MCS mini culotte stenting; MRC — mono-ring culotte stenting; NSTEMI — non-ST elevation myocardial infarction; OMs - obtuse marginal branches; $\mathrm{PCl}$ - percutaneous coronary intervention; PDA - posterior descending artery; PLA — posterolateral artery; RCA — right coronary artery; STEMI - ST elevation myocardial infarction

for propensity score matching. Finally, 21 patients treated by MRC were matched with 42 patients by MCS.

\section{Baseline characteristics}

There were no differences in the baseline clinical characteristics between the two groups neither before nor after propensity score matching. As for baseline lesion characteristics, BDD of the MB and SB was bigger $(0.68 \pm 0.41 \mathrm{~mm}$ vs. $0.51 \pm 0.31 \mathrm{~mm}$, $\mathrm{p}=0.025)$ and the average SYNTAX score was significantly lower $(20.7 \pm 7.1$ vs. $25.5 \pm 10.1, \mathrm{p}=0.031)$ in the MRC group than in the MCS group. Addi- tionally, there was a trend of more patients with Medina type $(1,1,1)$ lesions in the MRC group ( $87.0 \%$ vs. $62.2 \%$ in the MCS group, $\mathrm{p}=0.085$ ). After propensity score matching, no difference was observed in each lesion variable (Table 2).

\section{Procedural data}

Before propensity score matching, there were no differences in interventional characteristics between the two groups except that the stent length of $\mathrm{MB}$ and $\mathrm{SB}$ was shorter in the MRC group than in the MCS group (MB: $27.7 \pm 10.8 \mathrm{~mm}$ vs. $37.6 \pm$ $\pm 16.1 \mathrm{~mm}, \mathrm{p}=0.005$; SB: $15.0 \pm 6.2 \mathrm{~mm}$ vs. 
$21.1 \pm 9.6 \mathrm{~mm}, \mathrm{p}=0.004)$. In the MCS group, fKBD was not complete in 2 patients and slow TIMI flow of SB occurred in 2 patients. No stent dislodgement occurred and fKBD was complete in all patients in the MRC group. Both stenting techniques had similarly high rate of device, angiographic, and procedural success. No death, target vessel/ /lesion revascularization or acute stent thrombosis were observed in the MRC group. Only 4 (17.4\%) patients had cTnT/cTnI $>5$ times of upper reference limit, but all of them had no ischemic chest pain and/or new onset of pathological $\mathrm{Q}$ waves. Correspondently, there were $3(2.5 \%)$ patients with non-Q-wave myocardial infarction and a trend of more patients $(24.4 \%)$ with $\mathrm{cTnT} / \mathrm{cTnI}>5$ times of upper reference limit in the MCS group. Death was observed in 1 patient from the MCS group, who was an 81-year-old male with a history of ischemia cardiomyopathy and low ejection fraction (21\%); he died of acute heart failure 7 days after procedure. There was no difference in the in-hospital major cardiac adverse events between the two groups. Furthermore, MRC resulted in shorter procedural $(33.4 \pm 9.1 \mathrm{~min}$ vs. $45.7 \pm 19.5 \mathrm{~min}, \mathrm{p}=0.004)$ and fluoroscopic time $(19.3 \pm 5.0 \mathrm{~min}$ vs. $25.1 \pm$ \pm 8.9 min, $p=0.003$ ), and less contrast media use $(113.0 \pm 29.3 \mathrm{~mL}$ vs. $155.6 \pm 71.3 \mathrm{~mL}, \mathrm{p}=0.006)$ when compared with the other group.

After propensity score matching, all of technical parameters were adjusted. There were no differences in device, angiographic and procedural success, in-hospital major cardiac adverse events, or even patients with cTnT/cTnI $>5$ times of upper reference limit. However, the advantage was that MRC reducing procedural $(33.3 \pm 9.5$ min vs. $46.7 \pm$ $\pm 15.6 \mathrm{~min}, \mathrm{p}=0.001)$ and fluoroscopic time (19.7 \pm \pm 4.9 min vs. $26.2 \pm 7.1 \mathrm{~min}, \mathrm{p}<0.001$ ), and less contrast media $(114.3 \pm 28.9 \mathrm{~mL}$ vs. $156.5 \pm 56.4 \mathrm{~mL}$, $\mathrm{p}=0.002$ ) was still preserved when compared with the MCS (Table 3).

\section{QCA data}

Before propensity score matching, imbalance was obvious between the two groups. At baseline, the lesion length of MV and SB were shorter in the MRC group than in the MCS group (MV: $27.0 \pm$ $\pm 11.3 \mathrm{~mm}$ vs. $34.1 \pm 15.3$, $\mathrm{p}=0.038$; SB: $13.1 \pm$ $\pm 5.7 \mathrm{~mL}$ vs. $18.5 \pm 9.6 \mathrm{~mL}, \mathrm{p}=0.010$ ). For the $\mathrm{SB}$ of MCS, especially minimal lumen diameter was smaller $(0.59 \pm 0.42 \mathrm{~mm}$ vs. $0.78 \pm 0.33 \mathrm{~mm}$, $\mathrm{p}=0.044$ ) and diameter stenosis was more severe $(77.6 \% \pm 14.8 \%$ vs. $70.6 \% \pm 12.1 \%, \mathrm{p}=0.034)$ than those of MRC. Post-procedurally, both the diameter stenosis of $\mathrm{MV} / \mathrm{MB}(6.6 \% \pm 2.7 \%$ vs.
$8.8 \% \pm 4.0 \%, \mathrm{p}=0.010)$ and $\mathrm{SB}(10.1 \% \pm 5.3 \%$ vs. $19.3 \% \pm 11.4 \%, \mathrm{p}<0.001)$ in the MRC group were lower than those in the MCS group. After propensity score matching, imbalance was adjusted among baseline quantitative analysis between the two groups. Post-procedurally, the diameter stenosis of MV/MB in the MRC group $(7.8 \% \pm$ $\pm 5.9 \%$ ) was similar to that in the MCS group $(9.1 \% \pm 5.1 \%, p=0.382)$. However, by comparison with the MCS, the MRC significantly reduced the diameter stenosis of SB $(8.7 \% \pm 11.0 \%$ vs. $16.8 \% \pm$ $\pm 11.2 \%, \mathrm{p}=0.008)$ (Table 4).

\section{Discussion}

An ideal 2-stent technique should be able to restore the bifurcation as close as possible to the native one, along with technical simplicity and procedural safety and efficacy. Our study demonstrated that MRC was associated not only with better bifurcated stent expansion and coverage at the cost of minimal strut overlap in bench testing but also less procedural complexity and ostial SB residual stenosis in comparison with MCS in the case-controlled study.

\section{What is new for MRC: Comparing with other related techniques}

Mono-ring culotte stenting is different from Szabo or the flower petal techniques mainly in ex vivo wiring of the most proximal stent cell [11-18]. The original Szabo or modified flower petal techniques utilized partial inflation of the SB stent to make a 'flower petal' and then to create a dual-wire stent-balloon unit prior to insertion. This step requires creating a bulkier dual-wire stent-balloon unit and weakening the stent fixation. The former will impede subsequent advancement of the device and the latter cause stent dislodgement due to frictional drag or wire entanglement [13-15]. Conversely, MRC allows to wire ex vivo only one of the most proximal cells with the hard end of the MB wire instead of partially inflating the whole stent, thus preserving the stent fixation, and to create a simpler and smaller dual-wire stent system rather than a more complex and larger dual-wire balloon-stent unit, thereby making the device advancement much easier with lower risk of stent dislodgement.

Recently, Toth et al. [19] reported a single string technique that provided several unique advantages, especially minimal overlapping strut that other culotte stenting techniques did not possess. For this technique, the first critical step is accurate positioning of the SB stent to ensure 
Table 3. Procedural data.

\begin{tabular}{|c|c|c|c|c|c|c|}
\hline \multirow[t]{2}{*}{ Variables } & \multicolumn{3}{|c|}{ Before propensity score matching } & \multicolumn{3}{|c|}{ After propensity score matching } \\
\hline & $\begin{array}{c}\text { MRC } \\
(n=23)\end{array}$ & $\begin{array}{c}\text { MCS } \\
(n=119)\end{array}$ & $\mathbf{P}$ & $\begin{array}{c}\text { MRC } \\
(n=21)\end{array}$ & $\begin{array}{c}\text { MCS } \\
(n=42)\end{array}$ & $\mathbf{P}$ \\
\hline Trans-radial & $20(87.0 \%)$ & $94(80.0 \%)$ & 0.658 & $20(95.2 \%)$ & $39(92.9 \%)$ & 1.000 \\
\hline Sheath size: & & & 0.495 & & & 0.832 \\
\hline $6 \mathrm{~F}$ & $5(21.7 \%)$ & $22(18.5 \%)$ & & $5(23.8 \%)$ & $8(19.0 \%)$ & \\
\hline $7 \mathrm{~F}$ & $18(78.3 \%)$ & $89(74.8 \%)$ & & $16(76.2 \%)$ & $33(78.6 \%)$ & \\
\hline $8 \mathrm{~F}$ & $0(0.0 \%)$ & $8(6.7 \%)$ & & $0(0.0 \%)$ & $1(2.4 \%)$ & \\
\hline \multicolumn{7}{|l|}{ Pre-dilation: } \\
\hline $\mathrm{MB} / \mathrm{MV}$ & $16(69.6 \%)$ & $63(52.9 \%)$ & 0.142 & $14(66.7 \%)$ & $27(64.3 \%)$ & 1.000 \\
\hline SB & $9(39.1 \%)$ & $45(37.8 \%)$ & 0.905 & $8(38.1 \%)$ & $16(38.1 \%)$ & 1.000 \\
\hline \multicolumn{7}{|l|}{ Post-dilation*: } \\
\hline $\mathrm{MB} / \mathrm{MV}$ & $23(100.0 \%)$ & $119(100.0 \%)$ & 1.000 & $21(100.0 \%)$ & $42(100.0 \%)$ & 1.000 \\
\hline SB & $23(100.0 \%)$ & $117(98.3 \%)$ & 1.000 & $21(100.0 \%)$ & $41(97.6 \%)$ & 1.000 \\
\hline Final kissing balloon dilation* & $23(100 \%)$ & $117(98.3 \%)$ & 1.000 & $21(100.0 \%)$ & $41(97.6 \%)$ & 1.000 \\
\hline \multicolumn{7}{|l|}{ Stent diameter: } \\
\hline $\mathrm{MB} / \mathrm{MV}$ & $3.18 \pm 0.51$ & $3.14 \pm 0.48$ & 0.654 & $3.23 \pm 0.51$ & $3.19 \pm 0.51$ & 0.788 \\
\hline SB & $2.71 \pm 0.39$ & $2.88 \pm 0.44$ & 0.087 & $2.73 \pm 0.40$ & $2.80 \pm 0.35$ & 0.436 \\
\hline \multicolumn{7}{|l|}{ Total stent length [mm]: } \\
\hline $\mathrm{MB} / \mathrm{MV}$ & $27.7 \pm 10.8$ & $37.6 \pm 16.1$ & 0.005 & $28.5 \pm 10.8$ & $34.1 \pm 17.3$ & 0.184 \\
\hline SB & $15.0 \pm 6.2$ & $21.1 \pm 9.6$ & 0.004 & $15.1 \pm 6.4$ & $16.2 \pm 4.9$ & 0.464 \\
\hline \multicolumn{7}{|c|}{$>20 \%$ of residual diameter stenosis*: } \\
\hline $\mathrm{MB} / \mathrm{MV}$ & $0(0 \%)$ & $0(0 \%)$ & 1.000 & $0(0 \%)$ & $0(0 \%)$ & 1.000 \\
\hline SB & $0(0 \%)$ & $2(1.7 \%)$ & 1.000 & $0(0 \%)$ & $1(2.4 \%)$ & 1.000 \\
\hline \multicolumn{7}{|l|}{$<3$ of TIMI flow*: } \\
\hline $\mathrm{MB} / \mathrm{MV}$ & $0(0 \%)$ & $0(0.8 \%)$ & 1.000 & $0(0 \%)$ & $0(0 \%)$ & 1.000 \\
\hline SB & $0(0 \%)$ & $2(1.7 \%)$ & 1.000 & $0(0 \%)$ & $0(0 \%)$ & 1.000 \\
\hline \multicolumn{7}{|l|}{ Success rate*: } \\
\hline Technical & $23(100.0 \%)$ & $116(97.5 \%)$ & 1.000 & $21(100 \%)$ & $41(97.6 \%)$ & 1.000 \\
\hline Angiographic & $23(100.0 \%)$ & $117(98.3 \%)$ & 1.000 & $21(100 \%)$ & $41(97.6 \%)$ & 1.000 \\
\hline Procedural & $23(100.0 \%)$ & $114(95.8 \%)$ & 1.000 & $21(100 \%)$ & $40(95.2 \%)$ & 0.548 \\
\hline \multicolumn{7}{|c|}{ Periprocedural myocardial infarction*: } \\
\hline Non-Q-wave* & $0(0 \%)$ & $3(2.5 \%)$ & 1.000 & $0(0 \%)$ & $1(2.4 \%)$ & 1.000 \\
\hline Q-wave* & $0(0 \%)$ & $0(0 \%)$ & 1.000 & $0(0 \%)$ & $0(0 \%)$ & 1.000 \\
\hline $\begin{array}{l}\mathrm{cTnT} / \mathrm{cTnl}>5 \times \text { upper } \\
\text { reference limit* }\end{array}$ & $4(17.4 \%)$ & $29(24.4 \%)$ & 0.595 & $4(19.0 \%)$ & $9(21.4 \%)$ & 1.000 \\
\hline Acute in-stent thrombosis* & $0(0 \%)$ & $0(0 \%)$ & 1.000 & $0(0 \%)$ & $0(0 \%)$ & 1.000 \\
\hline $\begin{array}{l}\text { In-hospital major adverse } \\
\text { cardiac events* }\end{array}$ & $0(0 \%)$ & $4(3.4 \%)$ & 1.000 & $0(0 \%)$ & $1(2.4 \%)$ & 1.000 \\
\hline Procedural time ${ }^{\S}[\mathrm{min}]$ & $33.4 \pm 9.1$ & $45.7 \pm 19.5$ & 0.004 & $33.3 \pm 9.5$ & $46.7 \pm 15.6$ & 0.001 \\
\hline Total fluoroscopy time [min] & $19.3 \pm 5.0$ & $25.1 \pm 8.9$ & 0.003 & $19.7 \pm 4.9$ & $26.2 \pm 7.1$ & $<0.001$ \\
\hline Contrast volume [mL] & $113.0 \pm 29.3$ & $155.6 \pm 71.3$ & 0.006 & $114.3 \pm 28.9$ & $156.5 \pm 56.4$ & 0.002 \\
\hline
\end{tabular}

*Fisher's exact test; §Indicates the time from "guidewire in" to "guidewire out"; cTnT/cTnl — cardiac troponin T/cardiac troponin I; MB — main branch; MCS - mini culotte stenting; MRC — mono-ring culotte stenting; MV — main vessel; SB — side branch; TIMI — Thrombolysis in Myocardial Infarction

minimal protrusion into the MV, which is difficult due to complex bifurcation anatomy and continuous heart beating. Even if accurate position of the
SB stent is achieved, subsequent rewiring is still problematic due to the bifurcated anatomy and lesion complexity. Additionally, in vivo rewiring 
Table 4. Quantitative coronary analysis at baseline and immediate post-procedurally.

\begin{tabular}{|c|c|c|c|c|c|c|}
\hline \multirow[t]{2}{*}{ Variables } & \multicolumn{3}{|c|}{$\mathrm{MV} / \mathrm{MB}$} & \multicolumn{3}{|c|}{ SB } \\
\hline & MRC & MCS & $\mathbf{P}$ & MRC & MCS & $\mathbf{P}$ \\
\hline \multicolumn{7}{|c|}{ Before propensity score matching ( $n=23$ in group MRC; $n=119$ in group MCS) } \\
\hline \multicolumn{7}{|l|}{ Baseline } \\
\hline Lesion length $[\mathrm{mm}]$ & $27.0 \pm 11.3$ & $34.1 \pm 15.3$ & 0.038 & $13.1 \pm 5.7$ & $18.5 \pm 9.6$ & 0.010 \\
\hline $\begin{array}{l}\text { Reference vessel } \\
\text { diameter }[\mathrm{mm}]\end{array}$ & $3.35 \pm 0.57$ & $3.15 \pm 0.49$ & 0.078 & $2.67 \pm 0.39$ & $2.63 \pm 0.44$ & 0.708 \\
\hline $\begin{array}{l}\text { Minimal lumen } \\
\text { diameter [mm] }\end{array}$ & $0.72 \pm 0.35$ & $0.54 \pm 0.56$ & 0.153 & $0.78 \pm 0.33$ & $0.59 \pm 0.42$ & 0.044 \\
\hline Diameter stenosis [\%] & $78.1 \pm 11.2$ & $83.2 \pm 16.2$ & 0.149 & $70.6 \pm 12.1$ & $77.6 \pm 14.8$ & 0.034 \\
\hline \multicolumn{7}{|l|}{ Post-procedure } \\
\hline $\begin{array}{l}\text { Reference vessel } \\
\text { diameter [mm] }\end{array}$ & $3.44 \pm 0.64$ & $3.27 \pm 0.51$ & 0.163 & $2.78 \pm 0.44$ & $2.85 \pm 0.43$ & 0.462 \\
\hline $\begin{array}{l}\text { Minimal lumen } \\
\text { diameter [mm] }\end{array}$ & $3.21 \pm 0.58$ & $2.99 \pm 0.51$ & 0.058 & $2.50 \pm 0.42$ & $2.31 \pm 0.49$ & 0.084 \\
\hline Diameter stenosis [\%] & $6.6 \pm 2.7$ & $8.8 \pm 4.0$ & 0.010 & $10.1 \pm 5.3$ & $19.3 \pm 11.4$ & $<0.001$ \\
\hline \multicolumn{7}{|c|}{ After propensity score matching ( $n=21$ in group MRC; $n=42$ in group MCS) } \\
\hline \multicolumn{7}{|l|}{ Baseline } \\
\hline Lesion length $[\mathrm{mm}]$ & $27.6 \pm 11.2$ & $30.5 \pm 16.1$ & 0.464 & $13.4 \pm 5.9$ & $13.5 \pm 5.2$ & 1.000 \\
\hline $\begin{array}{l}\text { Reference vessel } \\
\text { diameter }[\mathrm{mm}]\end{array}$ & $3.44 \pm 0.62$ & $3.25 \pm 0.55$ & 0.209 & $2.69 \pm 0.33$ & $2.60 \pm 0.44$ & 0.407 \\
\hline $\begin{array}{l}\text { Minimal lumen } \\
\text { diameter [mm] }\end{array}$ & $0.70 \pm 0.32$ & $0.69 \pm 0.55$ & 0.934 & $0.76 \pm 0.34$ & $0.70 \pm 0.41$ & 0.571 \\
\hline Diameter stenosis [\%] & $79.6 \pm 10.4$ & $78.1 \pm 17.4$ & 0.713 & $71.7 \pm 11.8$ & $72.5 \pm 15.5$ & 0.832 \\
\hline \multicolumn{7}{|l|}{ Post-procedure } \\
\hline $\begin{array}{l}\text { Reference vessel } \\
\text { diameter [mm] }\end{array}$ & $3.48 \pm 0.61$ & $3.31 \pm 0.50$ & 0.243 & $2.77 \pm 0.42$ & $2.76 \pm 0.33$ & 0.951 \\
\hline $\begin{array}{l}\text { Minimal lumen } \\
\text { diameter [mm] }\end{array}$ & $3.19 \pm 0.52$ & $3.02 \pm 0.53$ & 0.218 & $2.52 \pm 0.44$ & $2.31 \pm 0.49$ & 0.111 \\
\hline Diameter stenosis [\%] & $7.8 \pm 5.9$ & $9.1 \pm 5.1$ & 0.382 & $8.7 \pm 11.0$ & $16.8 \pm 11.2$ & 0.008 \\
\hline
\end{tabular}

MB - main branch; MCS — mini-culotte stenting; MRC — mono-ring culotte stenting; MV — main vessel; SB — side branch

of a single cell needs an experienced hand and may dissect the MB or enlarge the MB dissection created by pre-dilation, or even cause disastrous MB occlusion. Unlike the single string technique, MRC wires the last stent cell ex vivo, which is surprisingly easy (Supplementary Video 1 - see journal website). Importantly, ex vivo wiring can reliably avoid the problems associated with in vivo wiring, leading to less technical complexity and better procedural safety.

\section{How to properly perform MRC:}

Tricks and tips

As shown in Figure 1, MRC was characterized by ex vivo wiring of the last proximal cell of the SB stent as an anchor to optimize stent protrusion into the MV. There are three key steps for the procedure: ex vivo wiring and anchoring of a proximal cell of the SB stent, inserting the dualwire stent unit into the target site and optimizing the SB stent protrusion and resultant bifurcated stent configuration.

Ex vivo wiring and anchoring of the most proximal stent cell can be readily achieved by using the hard end of the MB wire. We recommend this step being done by a younger interventionist with good eyesight. Advancing of the dual-wire stent unit into the target site may be somehow difficult. Lower or higher resistance will be encountered during device insertion; occasionally there is great difficulty in advancing the unit mainly due to wire entanglement. It is noteworthy that any rough action should be abandoned in processes of ex vivo wiring of the most proximal stent cell and push- 
ing the SB stent as it may result in stent damage and destroy the stent fixation so that the SB stent dislodges. To avoid such situations, selecting a bigger guiding catheter, full flaring of the proximal cell, using a hydrophilic coated and softer wire, avoiding too much wire rotation and separating the two twisted wires with a predilating balloon prior to SB stenting, or fully predilating of lesions, will be helpful. Actually, optimizing the stent protrusion and configuration is composed of two vital steps - minimizing stent protrusion into the MV and maintaining the ostial SB stent configuration without distortion. Anchoring the proximal cell of the SB stent automatically leads to minimizing the SB stent protrusion, while performing a sequential iKBD is essential for preventing the ostial SB stent from distortion, which can be done by first inflation of the SB balloon with higher pressure to fix the ostial SB stent, followed by inflation of the $\mathrm{MB}$ balloon with lower pressure to fully open the anchored cell.

\section{Outcomes of MRC: Comparing with MCS}

Culotte-based stenting techniques, particularly MCS, have regained enthusiasm in intervention of coronary bifurcation lesions because of several modifications that enhance the procedural safety and efficacy. Nevertheless, these techniques remain technically complex. By owning 3 key technical modifications, whether MRC can more effectively solve the problems associated with culotte-based stenting and translate into improvement of clinical outcomes warrants to be further tested.

In the present study, as compared to MCS, bench testing showed that MRC was associated with better stent configuration in terms of stent expansion and coverage in polygon of confluence and adjacent area (SB ostium, MB ostium, or carina). Meanwhile, the clinical case-controlled study also demonstrated that MRC not only had the same high rate of angiographic and procedural success as MCS but also effectively avoided stent advancing difficulty and stent dislodgement, as using Szabo and its modified technique, and technical challenges for in vivo wiring a single cell, as using single string technique [11-19]. Importantly, MRC significantly reduced procedural time, fluoroscopic time and contrast consumption, as well as residual stenosis of the ostial SB with good procedural safety.

\section{Limitations of the study}

In bench testing, despite considering greater $\mathrm{BDD}$ between $\mathrm{MB}$ and $\mathrm{SB}$, a silicon model may not exactly represent anatomy of coronary bifurcation lesions in clinical scenarios, therefore the findings should be explained with caution. In a clinical case-controlled study, although a propensity score matching was used to adjust anatomic differences of coronary bifurcation lesions, we cannot utterly exclude other hidden confounders (i.e. physician's preference), and only a small number of case pairs with short-term outcomes were included into analysis, which also cannot completely avoid bias. Therefore, a controlled, randomized, larger population study is warranted for further confirming the long-term efficacy of MRC.

\section{Conclusions}

In conclusion, as compared to MCS, our study demonstrated that MRC was associated with better bifurcated stent configuration in bench testing and less procedural complexity, as well as residual ostial stenosis of the SB in clinical application, indicating that ex vivo mono-ring technique was feasible for simplification of culotte stenting.

Grants: National natural science foundation of China (No. 81370311) and the Key Program of Social Development of Fujian Science and Technology Department (Grant No. 2013Y0043).

\section{Conflict of interest: None declared}

\section{References}

1. Chevalier B, GlattB, Royer T, Guyon P.Placement of coronary stents in bifurcation lesions by the "culotte" technique. Am J Cardiol, 1998; 82: 943-949.

2. Chen LL, Fan L, Chen ZY et al. Modified culotte stenting for treatment of complex coronary bifurcation lesions: Immediate and 9-month outcomes in a pilot study. Chin Med J, 2011; 124: 1943-1950.

3. Wen S, Yu H, Lee H. Mini-culotte stenting for bifurcation coronary disease. Chin Med J, 2014; 127: 978-979.

4. Fan L, Chen LL, Luo YK et al. DK mini-culotte stenting in the treatment of true coronary bifurcation lesions: A propensity score matching comparison with T-provisional stenting. Heart Vessels, 2016; 31: 308-321.

5. Ormiston JA, Webster MW, Stewart JT, Ruygrok PN, Hatrick RI. The "Crush" technique for coronary artery bifurcation stenting: insights from micro-computed tomographic imaging of bench deployments. J Am Coll Cardiol Cardiovasc Interv, 2008; 1: 351-357.

6. Lefevre T, Louvard Y, Morice MC et al. Stenting of bifurcation lesions: Classification, treatments, and results. Catheter Cardiovasc Interv, 2000;49: 274-283.

7. Medina A, Suarez de Lezo J, Pan M. A new classification of coronary bifurcation lesions. Rev Esp Cardiol, 2006; 59: 183. 
8. Erglis A, Kumsars I, Niemelä M et al.; Nordic PCI Study Group. Randomized comparison of coronary bifurcation stenting with the crush versus the culotte technique using sirolimus eluting stents: The Nordic stent technique study. Circ Cardiovasc Interv, 2009; 2: 27-34.

9. Levine GN, Bates ER, Blankenship JC et al. 2011 ACCF/AHA/ /SCAI Guideline for Percutaneous Coronary Intervention: A report of the American College of Cardiology Foundation/American Heart Association Task Force on Practice Guidelines and the Society for Cardiovascular Angiography and Interventions. Circulation, 2011; 124: e574-e651.

10. Cutlip DE, Windecker S, Mehran R et al.; Academic Research Consortium. Clinical end points in coronary stent trials: A case for standardized definitions. Circulation, 2007; 115: 2344-2351.

11. Kern MJ, Ouellette D, Frianeza T. A new technique to anchor stents for exact placement in ostial stenoses: The stent tail wire or Szabo technique. Catheter Cardiovasc Interv, 2006; 68: 901-906.

12. Gutiérrez-Chico JL, Villanueva-Benito I, Villanueva-Montoto L et al. Szabo technique versus conventional angiographic placement in bifurcations 010-001 of Medina and in aorto-ostial stenting: Angiographic and procedural results. EuroIntervention, 2010; 5: 801-808.
13. Jain RK, Padmanabhan TN, Chitnis N. Causes of failure with Szabo technique: An analysis of nine cases. Indian Heart J, 2013; 65: 264-268.

14. Ferrer-Gracia MC, Sánchez-Rubio J, Calvo-Cebollero I. Stent dislodgement during Szabo technique. Int J Cardiol, 2011; 147: e8-e9.

15. Vaquerizo B, Serra A, Ormiston J et al. Bench top evaluation and clinical experience with the Szabo technique: New questions for a complex lesion. Catheter Cardiovasc Interv, 2012; 79: 378-389.

16. Kinoshita $Y$, Katoh $O$, Matsubara $T$ et al. First clinical experience of "flower petal stenting": A novel technique for the treatment of coronary bifurcation lesions. J Am Coll Cardiol Cardiovasc Interv, 2010; 3: 58-65.

17. Çayl M, Şahin DY, Elbasan $Z$ et al. Modified flower petal technique: A new technique for the treatment of Medina type 1.1.1 coronary bifurcation lesions. J Am Coll Cardiol Cardiovasc Interv, 2013; 6: 516-522.

18. Colombo A, Latib A. The artisan approach for stenting bifurcation lesions. J Am Coll Cardiol Cardiovasc Interv, 2010; 3: 66-67.

19. Toth G, Pyxaras S, Mortier P et al. Single string technique for coronary bifurcation stenting: Detailed technical evaluation and feasibility analysis. J Am Coll Cardiol Cardiovasc Interv, 2015; 8: 949-959. 